\title{
Associations between Measures of Structural Morphometry and Sensorimotor Performance in Individuals with Nonspecific Low Back Pain
}

\author{
(D). Caeyenberghs, (D) M. Pijnenburg, (D) N. Goossens, DL. Janssens, and DS. Brumagne
}

\begin{abstract}
BACKGROUND AND PURPOSE: To date, most structural brain imaging studies in individuals with nonspecific low back pain have evaluated volumetric changes. These alterations are particularly found in sensorimotor-related areas. Although it is suggested that specific measures, such as cortical surface area and cortical thickness, reflect different underlying neural architectures, the literature regarding these different measures in individuals with nonspecific low back pain is limited. Therefore, the current study was designed to investigate the association between the performance on a sensorimotor task, more specifically the sit-to-stand-to-sit task, and cortical surface area and cortical thickness in individuals with nonspecific low back pain and healthy controls.
\end{abstract}

MATERIALS AND METHODS: Seventeen individuals with nonspecific low back pain and 17 healthy controls were instructed to perform 5 consecutive sit-to-stand-to-sit movements as fast as possible. In addition, T1-weighted anatomic scans of the brain were acquired and analyzed with FreeSurfer.

RESULTS: Compared with healthy controls, individuals with nonspecific low back pain needed significantly more time to perform 5 sit-to-stand-to-sit movements $(P<.05)$. Brain morphometric analyses revealed that cortical thickness of the ventrolateral prefrontal cortical regions was increased in patients with nonspecific low back pain compared with controls. Furthermore, decreased cortical thickness of the rostral anterior cingulate cortex was associated with lower sit-to-stand-to-sit performance on an unstable support surface in individuals with nonspecific low back pain and healthy controls $(r=-0.47, P<.007)$. In addition, a positive correlation was found between perceived pain intensity and cortical thickness of the superior frontal gyrus $(r=0.70, P<.002)$ and the pars opercularis of the inferior ventrolateral prefrontal cortex $(r=0.67, P<.004)$. Hence, increased cortical thickness was associated with increased levels of pain intensity in individuals with nonspecific low back pain. No associations were found between cortical surface area and the pain characteristics in this group.

CONCLUSIONS: The current study suggests that cortical thickness may contribute to different aspects of sit-to-stand-to-sit performance and perceived pain intensity in individuals with nonspecific low back pain.

ABBREVIATIONS: DASS-21 = Depression Anxiety Stress Scale; NPRS = numeric pain rating scale; NSLBP = nonspecific low back pain; ODI = Oswestry Disability Index; STSTS = sit-to-stand-to-sit

$\mathbf{N}$ onspecific low back pain (NSLBP) refers to low back pain that is not attributable to a specific cause. This category of low back pain disorders includes almost all low back pain symp-

Received January 5, 2015; accepted after revision August 18, 2016.

From the School of Psychology (K.C.), Faculty of Health Sciences, Australian Catholic University, Sydney, New South Wales, Australia; Department of Rehabilitation Sciences (M.P., N.G., L.J., S.B.), Katholieke Universiteit Leuven, Leuven, Belgium; and Hasselt University (L.J.), Biomedical Research Institute, Diepenbeek, Belgium.

K. Caeyenberghs and M. Pijnenburg are co-first authors.

This work was supported by the Agency for Innovation by Science and TechnologyFlanders (http://www.iwt.be) (PhD fellowship to Madelon Pijnenburg and PhD fellowship to Nina Goossens, grant No, 141037) and by the Research Foundation Flanders (http://www.fwo.be) (postdoctoral fellowship: Lotte Janssens, grant No. 12M9815N).

The funders had no role in study design; collection, analysis, and interpretation of data; in the writing of the manuscript; or in the decision to submit the manuscript for publication. toms. ${ }^{1-3}$ Despite much effort in the development of treatment strategies for this large population, ${ }^{4}$ the effects of current NSLBP interventions are rather small. Therefore, understanding the underlying neural basis of NSLBP is crucial.

Previous imaging studies showed structural alterations in cortical and subcortical brain regions in individuals with NSLBP. However, mixed findings were obtained. Both increases and decreases in gray matter volume in different brain

Please address correspondence to Madelon Pijnenburg, MD, Katholieke Universiteit Leuven, University of Leuven, Faculty of Kinesiology and Rehabilitation Sciences, Department of Rehabilitation Sciences, Research Group for Musculoskeletal Rehabilitation, Tervuursevest 101, Bus 1501 B-3001 Leuven; e-mail: m.pijnenburg@fontys.nl

- Indicates open access to non-subscribers at www.ajnr.org

三 Indicates article with supplemental on-line table.

http://dx.doi.org/10.3174/ajnr.A5020 
regions were found in individuals with NSLBP compared with healthy controls. Volumetric alterations in individuals with NSLBP were observed, for example, in the dorsolateral prefrontal cortex, ${ }^{5-7}$ in the somatosensory cortex, ${ }^{6,8,9}$ in the temporal lobes, ${ }^{6,8}$ and in the thalamus. ${ }^{5-7}$ Together, most gray matter alterations in NSLBP, either reduced or increased, are observed in areas related to sensorimotor control. These alterations in sensorimotor-related areas are indicative of impaired sensorimotor performance, as observed in individuals with NSLBP with behavioral measures. ${ }^{10-12}$ For example, individuals with NSLBP need notably more time to perform 5 consecutive sit-to-stand-to-sit (STSTS) movements compared with healthy controls. ${ }^{13}$ This STSTS task necessitates optimal sensorimotor control, which requires an efficient processing of sensory and motor information across the brain. ${ }^{14}$ However, nearly all structural brain imaging studies in NSLBP and in sensorimotor control have evaluated volumetric changes. ${ }^{5-9}$ Only 1 study in patients with Parkinson disease investigated how structural morphometry was associated with motor performance, showing an association between cortical thinning of the sensory parietotemporal areas and motor deficits. ${ }^{15}$ However, in NSLBP, cortical thickness and cortical surface area have been relatively understudied, while these 2 aspects of brain structure may be crucial to functional connectivity.

Cortical thickness and cortical surface area have a distinct genetic origin, ${ }^{16,17}$ a contrasting phylogeny, ${ }^{18}$ and different developmental trajectories. ${ }^{19}$ In addition, it is suggested that cortical thickness and cortical surface area reflect different aspects of the underlying neural architecture. ${ }^{20}$ More specifically, cortical surface area is primarily determined by the number of columns within a cortical region, whereas cortical thickness is thought to reflect the number of cells within these cortical columns. ${ }^{18,21}$ Therefore, evaluation of cortical surface area and cortical thickness as separate measures can provide interesting additional knowledge on the neural mechanisms of NSLBP and sensorimotor tasks. These measures of structural morphometry can be computed by a surface-based analysis method called FreeSurfer (http://surfer.nmr.mgh.harvard.edu). ${ }^{22}$

With the FreeSurfer analysis suite, 2 recent studies ${ }^{23,24}$ have provided evidence for alterations in cortical thickness in individuals with NSLBP compared with healthy controls. Although, Kong et $\mathrm{al}^{23}$ found increased cortical thickness in the bilateral primary somatosensory cortex, somatotopically associated with the lower back, in individuals with NSLBP, Dolman et al, ${ }^{24}$ demonstrated that the differences in cortical thickness between individuals with NSLBP and healthy controls disappeared when controlling for age. Nevertheless, little research has been done on the associations with sensorimotor control and pain by using both surface area and cortical thickness. Therefore, this study was designed to investigate the distinct relation between the STSTS performance and the cortical surface area and cortical thickness in individuals with NSLBP and healthy controls. An association between cortical thinning of sensorimotor brain areas and a longer duration to perform 5 consecutive STSTS movements was hypothesized. This correlation analysis was performed to reveal the potential different contributions of the 2 nonvolumetric parameters to sensorimotor control. In addition, considering recent findings, ${ }^{23,24}$ we hypothesized subtle cortical thinning in both sensorimotor- and pain-related brain regions in individuals with NSLBP compared with healthy controls.

\section{MATERIALS AND METHODS \\ Participants}

Thirty-four subjects were studied, including 17 subjects with NSLBP (11 women and 6 men) and 17 age-matched ( \pm 2 years) healthy individuals ( 12 women and 5 men). Data from the same cohort were previously reported. ${ }^{25,26}$ Subjects with NSLBP were recruited consecutively from 2012 to 2013 , as they responded to flyers in various settings (ie, academic [University Hospital Leuven] or community [sport clubs]), physician referrals (speciality care), mailings, and the Internet advertisements. Subjects with NSLBP were included under the following conditions: 1) they were between 20 and 50 years of age, 2) had experienced at least 6 months of disabling NSLBP (Oswestry Disability Index, Version 2 [adapted Dutch version, ODI-2] ${ }^{27}$ of $>12 \%$ ), 3) were not taking heavy opioids or drugs, 4) did not have vestibular and/or selfreported specific balance problems that precluded participation in the study procedure, 5) had no previous history of brain injury or other neurologic disorders, 6) had no neck problems (Neck Disability Index ${ }^{28}$ of $\left.<6 \%\right), 7$ ) had no previous major trauma and/or operation of the spine or lower limbs, and 8) met the standard "MR safety" bench test criteria (eg, no claustrophobia, no metal implants in body). Six left-handed participants (2 patients with NSLBP and 4 healthy controls) were included in the study; however, the removal of left-handed participants did not change the results on group differences in structural morphometry and correlation analyses. All participants gave their written informed consent before the study. The study conformed to the principles of the Declaration of Helsinki (1964) and its later amendments; was approved by the local Ethics Committee of Biomedical Sciences, University Hospital Leuven, Belgium (s53802); and was registered at www.clinicaltrails.gov with the identification number NCT01540617.

\section{Description of Measures}

Pain Characteristics. The pain characteristics are defined by the numeric pain rating scale (NPRS) of the back, scores on the ODI-2, and the number of years of NSLBP. The NPRS back pain scores ( 0 , no pain, to 10 , worst pain) during the last month $\left(\mathrm{NPRS}_{\text {back }}\right.$ usual) and at the moment of testing $\left(\mathrm{NPRS}_{\text {back }}\right.$ current) were reported. These scores are well-validated measures to define the intensity of NSLBP. ${ }^{29}$

Sit-to-Stand-to-Sit Task. The equipment, paradigm parameters, and dependent variables of the STSTS task were identical to those in previous studies. ${ }^{13,26}$ The participants were instructed to sit barefoot on a stool that was placed on a 6-channel force plate (Bertec, Columbus, Ohio) with their arms relaxed alongside their bodies and their vision occluded with nontransparent goggles. The stool height was adjusted for each participant to assure an angle of $90^{\circ}$ in both hips and knees. After 15 seconds of usual sitting (no instructions on posture were given), participants were asked to perform 5 consecutive STSTS movements with a full range of motion as fast as possible. An investigator stood near the 
participant to prevent actual falls. The force plate registered anteroposterior center-of-pressure displacements. The center-ofpressure displacements were sampled at $500 \mathrm{~Hz}$ by using a Micro1401 data acquisition system and Spike2 software (Cambridge Electronic Design, Cambridgeshire, UK). This protocol was performed with the feet placed on both a stable (force plate itself) and an unstable support surface (50-cm length $\times 41-\mathrm{cm}$ width $\times$ 6-cm thickness [Balance Pad Elite; Airex, Sins, Switzerland], on the force plate). The total duration of the 5 consecutive STSTS movements was calculated on the basis of the anteroposterior center-of-pressure displacement. The starting- and end-points of the task were defined by the mean value of the center of pressure during usual sitting before and after the task. The STSTS task has shown good test-retest reliability (intraclass correlation coefficient $=0.84-0.94) .{ }^{30,31}$

Depression Anxiety Stress Scale. Finally, a short version of the Depression Anxiety Stress Scale (DASS-21) was administered. ${ }^{32}$ This is a set of 3 self-report scales designed to measure the negative emotional states of depression, anxiety, and stress. Subjects are asked to use the 4-point severity/frequency scales to rate the extent to which they have experienced each state during the past week. Scores for depression, anxiety, and stress are calculated by summing the scores for the relevant items.

\section{MR Imaging Acquisition and Analysis}

MR images were acquired with a 3T Achieva scanner (Philips Healthcare, Best, the Netherlands) equipped with a 32-channel standard head coil. High-resolution whole-brain T1-weighted anatomic scans were obtained with a $3 \mathrm{D}$ turbo field echo sequence $\left(\right.$ voxel size $=0.98 \times 0.98 \times 1.2 \mathrm{~mm}^{3}, \mathrm{TR}=9.59 \mathrm{~ms}, \mathrm{TE}=4.6 \mathrm{~ms}$, flip angle $=8^{\circ}, 182$ coronal sections, FOV $=250 \times 250 \times 218$ $\mathrm{mm}^{3}$, and matrix $=256 \times 256 \mathrm{~mm}^{2}$ ). All T1-weighted anatomic scans were checked by a radiologist to assure that no brain lesions were present.

The structural images were analyzed with the FreeSurfer analysis suite, which is documented and freely available for downloading on-line. A technical description of the FreeSurfer procedures can be found in previous publications. ${ }^{33-42}$ The whole-brain analysis was performed with additional computing resources from the high-performance computing Tier1 cluster at the University of Ghent (http://ugent.be/hpc/en). The FreeSurfer analysis suite is a multistep procedure that consists of the following: 1) removal of nonbrain tissue by using a hybrid watershed/surface deformation procedure (skull stripping), ${ }^{42}$ 2) automated transformation to Talairach space, 3) subject-specific parcellation of the subcortical white matter and deep gray matter volumetric structures, ${ }^{36,43}$ and 4) calculation of cortical surface area and cortical thickness from all vertices within the 34 cortical parcellations per hemisphere. ${ }^{44}$ Results of each subject were carefully visually inspected to ensure the accuracy of the skull striping, segmentation, and cortical surface reconstruction. Where needed, the appropriate manual corrections were performed as explained by the FreeSurfer Tutorial (http://surfer.nmr.mgh.harvard.edu/fswiki/FsTutorial). In some datasets, it appeared that the skull strip left a lot of dura. However, it did not affect the surfaces following the gray and white matter borders. The averaged values across hemispheres were calculated and used in the statistical analysis to reduce the number of comparisons.
Table 1: Characteristics of the participants ${ }^{a}$

\begin{tabular}{lccc}
\multicolumn{1}{c}{ Characteristic } & $\begin{array}{c}\text { NSLBP Group } \\
(\boldsymbol{n}=\mathbf{1 7})\end{array}$ & $\begin{array}{c}\text { Healthy Group } \\
(\boldsymbol{n}=\mathbf{1 7})\end{array}$ & P Value \\
\hline Age $(\mathrm{yr})$ & $33.3 \pm 7.9$ & $31.8 \pm 8.2$ & .582 \\
Sex (male/female) & $6 / 11$ & $5 / 12$ & .724 \\
Height $(\mathrm{cm})$ & $173.0 \pm 6.4$ & $169.1 \pm 6.4$ & .087 \\
Weight $(\mathrm{kg})$ & $72.7 \pm 10.6$ & $64.9 \pm 10.2$ & .036 \\
BMI $\left(\mathrm{kg} / \mathrm{m}^{2}\right)$ & $24.2 \pm 2.8$ & $22.6 \pm 2.7$ & .090 \\
ODI-2 & $20.6 \pm 7.6$ & 0 & NA \\
Years of NSLBP & $9.8 \pm 8.2$ & 0 & NA \\
NPRS $_{\text {back }}$ usual & $4.5 \pm 2.0$ & 0 & NA \\
NPRS $_{\text {back }}$ current & $2.0 \pm 2.0$ & 0 & NA \\
\hline
\end{tabular}

Note:-BMI indicates body mass index; NA, not applicable.

${ }^{a}$ Data are presented as mean \pm SD. Significance level is $P<.05$.

\section{Statistical Analysis}

Unpaired $t$ tests were used to calculate group differences in characteristics of the participants. To determine whether an interaction effect of the Group $\times$ Support Surface was present in the STSTS performance, we performed a $2 \times 2$ repeated-measures ANOVA with Group as the between-subject factor (NSLBP group and healthy control group) and Support Surface as the withinsubject factor (stable support surface and unstable support surface). The significance level for group differences in characteristics and STSTS performance was set at $P<.05$. MANCOVA was used to contrast cortical thickness and surface area measurements from each cortical parcellation by Group while controlling for age. The Bonferroni correction was applied to correct for multiple comparisons, resulting in an adjusted $P$ value of $<.001(.05 / 34)$ for group differences in structural morphometry.

In addition, for each cortical parcellation from the morphologic measurement differences between groups, we also conducted post hoc power analyses by using $\mathrm{G}^{\star}$ Power, Version 3.1.9.2 (http://www.softpedia.com/get/Science-CAD/G-Power. shtml; with power $[1-\beta]$ set at 0.80 and $\alpha=.05,2$-tailed), to determine whether negative findings could be attributed to a limited sample size. The Outliers Labeling Rule (with a g-factor of $2.2)^{45}$ was applied to detect outliers from both the STSTS data and the structural morphometry parameters. These values were excluded pair-wise from the correlation analysis. Relationships between STSTS performance and pain scores on one hand and the characteristics of morphometry on the other hand were examined by using partial (controlling for age) Spearman correlations across the total group and within each of the groups (individuals with NSLBP and healthy controls) in regions with significant group differences only. The $P$ values reported for correlations were uncorrected for multiple comparisons with a statistical threshold of $P<.01$. These analyses, while showing a consistent trend, should be considered exploratory. The statistical analysis was performed with SPSS 22 (IBM, Armonk, New York).

\section{RESULTS}

\section{Characteristics of the Participants}

In accordance with the inclusion criteria, individuals with NSLBP reported scores above zero on the parameters of NSLBP-related disability and pain intensity (ODI-2, NPRS ${ }_{\text {back }}$ usual and NPRS back current), whereas all healthy individuals scored zero on these parameters (Table 1). No significant differences in demographic characteristics were found between the in- 
dividuals with NSLBP and healthy controls $(P>.05)$, except for weight (NSLBP, $72.7 \pm 10.6 \mathrm{~kg}$; healthy controls, $64.9 \pm 10.2 \mathrm{~kg}$; $P=.036$ ) (Table 1). According to the cutoff scores of the DASS21, 3 individuals with NSLBP showed moderate-to-severe depression, 4 individuals with NSLBP showed moderate-to-severe anxiety, and 5 individuals with NSLBP showed moderate-to-severe stress, whereas all controls scored zero on the 3 scales of the DASS- 21 .

\section{Sit-to-Stand-to-Sit Task}

A significant main effect of the factors Group $(F=11.348, P=$ $.002)$ and Surface $(F=6.29, P=.017)$ was observed in the duration of performing 5 consecutive STSTS movements. More specifically, individuals with NSLBP needed significantly more time to perform the STSTS task on stable and unstable support surfaces (stable, $18.1 \pm 6.9$ seconds; unstable, $16.8 \pm 5.9$ seconds) compared with healthy controls (stable, $12.9 \pm 2.5$ seconds; unstable, $11.5 \pm 2.0$ seconds $)(P=.002)$. Moreover, a significant decrease in the duration of the STSTS task was found on unstable support surfaces compared with the stable conditions, irrespective of Group $(P=.017)$. No interaction effect between the factors Group and Surface was present $(P>.05)$.

\section{Contribution of Weight}

Because a significant difference in weight was found between subjects with NSLBP and healthy controls, post hoc correlation anal-

Table 2: Relationships between weight and measures of pain

\begin{tabular}{lcc}
\multicolumn{1}{c}{ Measure of Pain } & $\begin{array}{c}\text { Spearman Correlation } \\
\text { Coefficient }\end{array}$ & $\boldsymbol{P}$ Value \\
\hline NPRS back $_{\text {Nacual }}$ current & 0.314 & .071 \\
NPRS $_{\text {back }}$ Curre & 0.323 & .630 \\
Years of NSLBP & 0.303 & .082 \\
No. of episodes of NSLBP & 0.357 & .038 \\
\hline
\end{tabular}

yses were performed to determine whether weight was associated with pain scores, structural morphometry measures, and STSTS performance. Our results revealed a significant positive correlation between the number of episodes of NSLBP and weight $(r=$ $0.357, P=.038$ ). No significant correlations between weight and the other measures of pain could be demonstrated (Table 2). Also, the weight of the participants was not significantly correlated with the total time needed to perform the STSTS task on stable and unstable support surfaces or with the structural morphometry $(P>.05)$. Therefore, weight was not included in the subsequent analysis.

\section{Structural Morphometry}

MANCOVA of the cortical parcellations between subjects with NSLBP and healthy controls (On-line Tables 1 and 2 and Fig 1), including age as a covariate in the model, showed cortical thickening in the individuals with NSLBP in the pars opercularis and pars triangularis of the inferior ventrolateral prefrontal cortex $(P<.001)$. These brain regions did retain significance after correcting for multiple comparisons. Using a less stringent threshold of $P<.01$ (indicated by footnote $a$ in On-line Table 1), we observed a cortical thickening trend in the NSLBP group in the cuneus $(P<.003)$, fusiform gyrus $(P<.004)$, inferior parietal gyrus $(P<.002)$, lateral orbitofrontal cortex $(P<.004)$, posterior cingulate gyrus $(P<.008)$, superior frontal gyrus $(P<.01)$, superior temporal gyrus $(P<.006)$, and transverse temporal gyrus $(P<$ $.004)$ and a cortical thinning trend in the rostral anterior cingulate gyrus $(P<.009)$ and the insula $(P<.002)$.

Within these regions, we checked for correlations to depression, anxiety, and stress (as measured by the DASS-21) to exclude the possibility that the significant group differences in cortical thickness may be explained by differences in emotional states. Only 1 significant positive correlation within the patient group

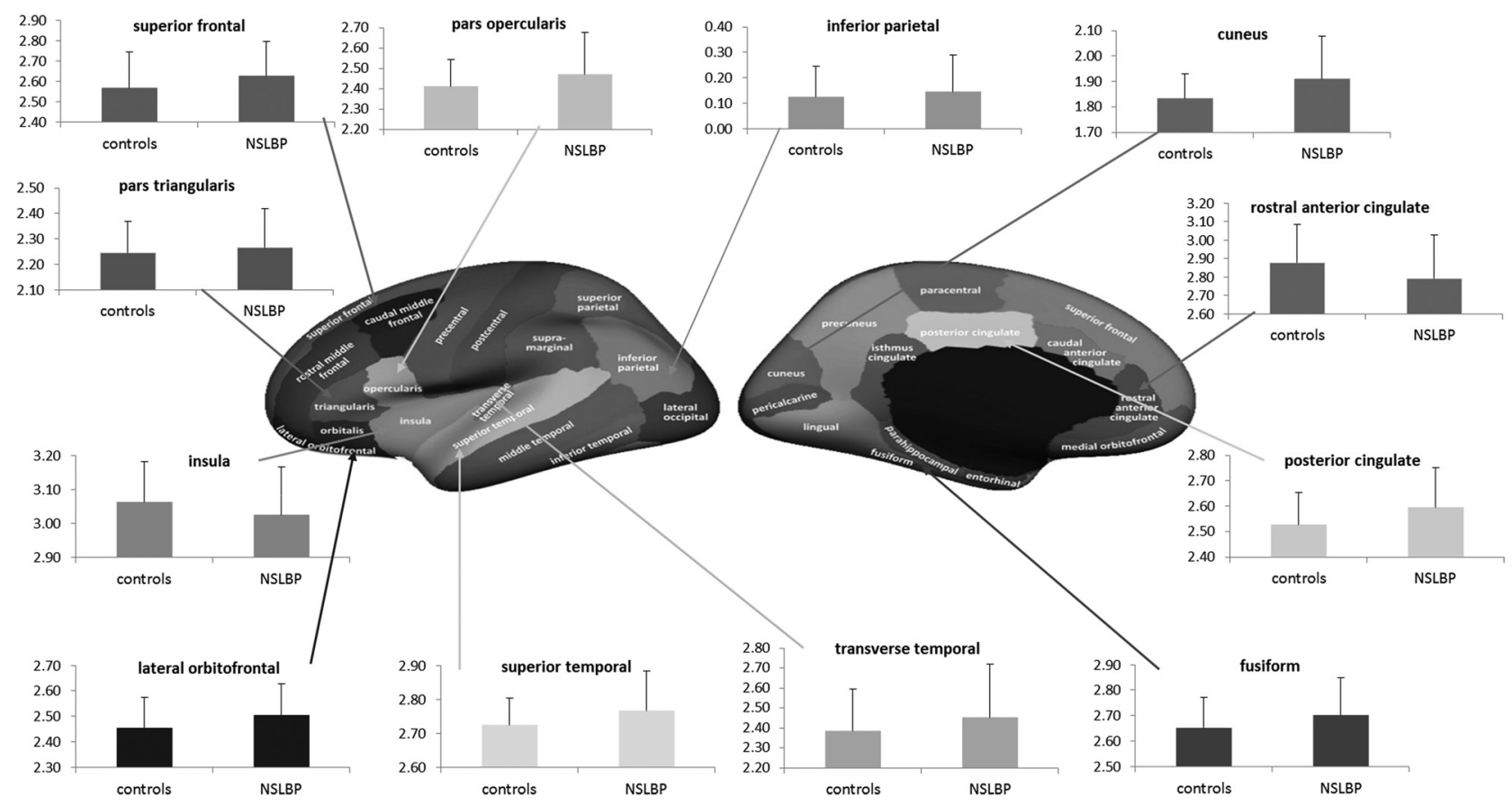

FIG 1. Significant group differences (after correcting for age) in cortical thickness. 

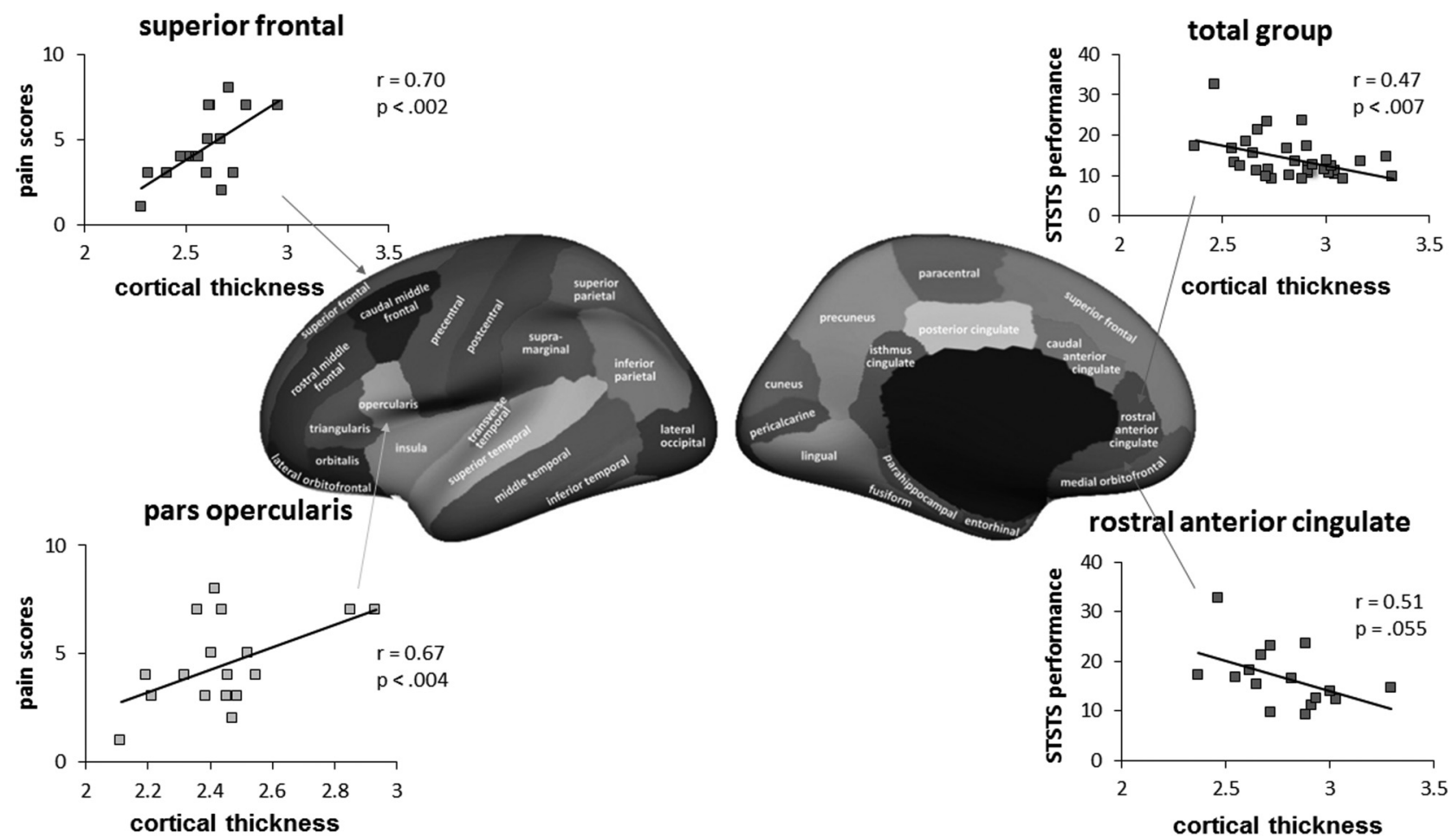

FIG 2. Scatterplots indicating the relationship between cortical thickness and STSTS performance and the pain-intensity score.

between the Depression Scale and cortical thickness was observed for the superior temporal gyrus $(r=0.66, P<.007)$.

No significant differences in cortical surface area between individuals with NSLBP and healthy controls could be demonstrated $(P>.001$, On-line Table 2$)$. On-line Table 2 also shows the sample sizes required to find statistically significant differences in surface area between the groups for each cortical parcellation. The numbers needed per group ranged from 41 to 29,790 . These results show that sample size would have to increase up to at least 41 for surface area measurements for group differences to reach statistical significance at the .05 level. Thus, it is likely that our negative findings for surface area can be attributed to a limited sample size.

\section{Association between Cortical Thickness and the Sit-to- Stand-to-Sit Performance}

Relationships (corrected for age) between STSTS performance and cortical thickness were investigated in regions with significant group effects. The duration to perform 5 consecutive STSTS movements on an unstable support surface was negatively correlated with the cortical thickness of the rostral anterior cingulate ( $r=-0.47, P<.007)$ within the total group (Fig 2). In other words, decreased cortical thickness of the rostral anterior cingulate cortex was associated with a longer duration of the STSTS task on an unstable support surface (lower performance). To some extent, this correlation coefficient between cortical thickness and STSTS performance was valid only for the NSLBP group ( $r=$ $-0.51, P=.055)$

\section{Association between Structural Morphometry and Pain Characteristics}

Within the group of individuals with NSLBP, significant positive correlations (as shown in Fig 2) were found between the NPRS back pain score (indexed by the $\mathrm{NPRS}_{\text {back }}$ usual) and the cortical thickness of the superior frontal gyrus $(r=0.70, P<.002)$ and the pars opercularis of the inferior ventrolateral prefrontal cortex $(r=0.67, P<.004)$. In other words, increased cortical thickness of the superior frontal gyrus and pars opercularis of the inferior ventrolateral prefrontal cortex was associated with an increased level of pain intensity in the individuals with NSLBP. No significant correlations were found between cortical surface area and the pain characteristics in this group of individuals with NSLBP.

\section{DISCUSSION}

This study is the first one correlating structural morphometrics with STSTS performance in patients with chronic pain, more specifically NSLBP. Brain morphometric analyses revealed that cortical thickness of the ventrolateral prefrontal cortical regions was increased in patients with NSLBP compared with controls. This increased cortical thickness was positively correlated with increased pain scores in the NSLBP group. Our behavioral results showed that individuals with NSLBP needed significantly more time to perform 5 consecutive STSTS movements on stable and unstable support surfaces. In addition, decreased cortical thickness of the rostral anterior cingulate cortex was associated with lower STSTS performance on unstable support surfaces.

\section{Increased Cortical Thickness in Ventrolateral Prefrontal Cortical Regions: Association with Pain Intensity}

Numerous studies with voxel-based morphometry have examined alterations of gray matter densities within specific brain regions in chronic pain conditions (for meta-analyses, see Pan et al, ${ }^{46}$ and Smallwood et al). ${ }^{47}$ However, surface-based features, such as cortical thickness and surface area, are more direct mea- 
sures of cortical morphometry than the gray matter density values used in voxel-based morphometry. ${ }^{34,48}$ To our knowledge, no study to date has examined these 2 measures in a group of individuals with chronic pain. So far, only 2 studies have investigated cortical thickness in individuals with NSLBP by using the FreeSurfer analysis suite. One study demonstrated an increased cortical thickness of the primary somatosensory cortex, more specifically the area somatotopically representing the lower back. ${ }^{23}$ In another study, group comparisons revealed cortical thickening in the right rostral middle frontal gyrus and a trend toward cortical thickening in the right paracentral lobule in patients with chronic low back pain. These regions did not retain significance after correcting for age. These previous findings of cortical thickening comport with our results. Our analyses, after correcting for age, revealed cortical thickening in individuals with NSLBP in the pars opercularis and pars triangularis, which together form the midportion of the ventrolateral prefrontal cortex. The changes identified in the ventrolateral prefrontal cortical regions in our group of subjects with NSLBP have face validity-that is, these brain regions appear to play an important role in the cognitive regulation of pain and emotion. ${ }^{49,50}$ Several voxel-based morphometry studies have identified changes in the ventrolateral prefrontal cortex in patients with other chronic pain conditions, such as migraine. ${ }^{51,52}$ Experimental pain studies with functional imaging techniques have also demonstrated altered activation of the same brain regions. ${ }^{53}$

The ventrolateral prefrontal cortex has also been reported to be involved in patients with anxiety, depressive symptoms, or stress. ${ }^{54,55}$ However, with the DASS-21, we checked whether the group results could be explained by depression, anxiety, or stress. We found only 1 significant positive correlation between cortical thickness of the superior temporal gyrus and the depression scale score. The present results convincingly show the important involvement of the ventrolateral prefrontal regions in pain processing. We have also found changes in cortical thickness in other structures known to be associated with pain processing and modulation, including the orbitofrontal regions, cingulate cortex, insula, and inferior parietal lobule. However, these regions did not survive Bonferroni correction.

Moreover, our results revealed that the pattern of specific alterations in brain morphology was directly related to the intensity of pain (ie, the increase in cortical thickness of the pars opercularis of the inferior frontal gyrus corresponded to greater pain). Correlation analyses revealed that increased cortical thickness was related to higher pain intensity scores in the NSLBP group. Our results are consistent with those in a previous study of SchmidtWilcke et al, 2006, ${ }^{6}$ in patients with chronic back pain in which brain regions showing an increase in gray matter density (such as the left thalamus and left putamen) coincided with increasing pain intensity.

Our findings of the relation between increased cortical thickness and increased pain scores in patients with NSLBP may reflect the consequence of a reorganization process of brain regions involved in a disproportionate number of signals of pain, emotion, and cognition. It can be suggested that this increased cortical thickness of the ventrolateral prefrontal cortex can eventually be normalized by specific and targeted training. ${ }^{56}$ Limited evidence available in the literature ${ }^{57,58}$ shows that treating chronic pain with cognitive behavioral therapy can lead to alterations in prefrontal brain regions and that the changes in these prefrontal regions correlate with clinical improvement. Whether morphologic changes can be normalized needs to be addressed in further research.

\section{Decreased Cortical Thickness in the Rostral Anterior Cingulate Cortex: Relationship with Sensorimotor Performance}

The changes in morphology correlated not only with pain scores but also with functional changes in sensorimotor control. Our behavioral results revealed that the NSLBP group required significantly more time to perform the STSTS task on both stable and unstable support surfaces compared with the healthy controls. This result is in agreement with previous studies. ${ }^{13,26}$ The STSTS task on an unstable support surface is ultimately challenging the sensorimotor system (including the proprioceptive system) because this condition requires additional reweighting of the proprioceptive signals due to the decreased reliability of proprioceptive signals from the ankle region. ${ }^{59}$ Proprioception can be defined as "the unconscious perception of movement and spatial orientation arising from stimuli within the body," ${ }^{60}$ and the unstable support surface forces the central nervous system to down-weight the less reliable ankle muscle proprioception and consequently to up-weight the proprioceptive input from more proximal segments, to provide optimal postural control. ${ }^{59,61}$ Nevertheless, a faster performance on an unstable support surface compared with the stable condition was observed. Because of the nonrandomized order of these conditions, this is possibly due to a learning effect. Despite this limitation, the individuals with NSLBP needed more time to perform 5 consecutive STSTS movements compared with controls, in both conditions.

This finding fits with previous ones. ${ }^{13}$ The decreased performance on a sensorimotor task in individuals with NSLBP, as represented by the increased duration of the STSTS task, fits within the findings of previous studies showing impaired sensorimotor control in individuals with NSLBP. ${ }^{10,62}$ Indeed, the STSTS task requires optimal sensorimotor control, for example, in terms of postural control. ${ }^{63}$ Recently, an association was observed between the proprioceptive reweighting capacity and microstructural integrity of the superior cerebellar peduncle in individuals with NSLBP. This finding suggests a neural basis for sensorimotor impairments. ${ }^{25}$ In this current study, the association between the sensorimotor STSTS task and structural morphometry in terms of cortical surface area and cortical thickness in individuals with NSLBP and healthy controls was investigated. Evaluation of these nonvolumetric parameters as separate measures, reflecting different aspects of the underlying neural architecture, is important in investigating what drives sensorimotor tasks.

In the present study, decreased cortical thickness of the rostral anterior cingulate cortex was associated with lower STSTS performance on unstable support surfaces. This correlation was significant within the total group, and a trend within the group of the patients with NSLBP was found. The anterior cingulate cortex is considered part of the general pain matrix ${ }^{64,65}$ and has been im- 
plicated in anticipation of pain and affective processing of pain. ${ }^{66}$ Structural alterations in the anterior cingulate cortex have been reported in a wide range of chronic pain conditions. ${ }^{46,47}$ The anterior cingulate cortex is also the brain region that most consistently shows activation in response to acute pain stimuli. ${ }^{66}$ To our knowledge, this is the first time that an association between the cortical thickness of the anterior cingulate cortex and performance on a sensorimotor task in individuals with NSLBP and healthy controls has been observed.

Of note, our statistical analyses (group comparisons and correlation analyses) revealed only significance for cortical thickness. In light of the radial unit hypothesis, ${ }^{18,67}$ our significant cortical thickness findings may reflect abnormalities in the number or size of the neuronal cell bodies within the cortical mini-columns of the ventrolateral prefrontal cortical regions in patients with NSLBP. In contrast, the absence of cortical surface area findings does not support individuals with NSLBP possibly having abnormal proliferation or decline in the number of cortical minicolumns. However, the power of our study was probably not enough to detect a significant group difference in surface area. While our sample size was similar to that in prior studies investigating differences in brain structure in patients with chronic pain, ${ }^{46,47}$ we also performed post hoc power analyses to determine whether negative findings for surface area could be attributed to the low sample size. We demonstrated that for most cortical parcellations, at least 41 subjects were required per group to observe significant differences in surface area. To precisely interpret these findings with respect to their functional significance, further research addressing the relationship between cerebral micro- and macrostructures as well as brain function is clearly necessary.

\section{Limitations}

The main shortcoming of our study was the small sample size, especially for the numerous correlations performed from the FreeSurfer output. Replication of the present morphologic findings with a larger sample is warranted. Moreover, future studies in a large number of participants should also stratify the groups by age rather than controlling for age in the statistical analyses. Another limitation of the present study pertains to the correlative nature of our study. The correlation coefficients computed between pain scores and brain morphometry provided us information about the nature of the relations between these variables but did not allow tests of strong causal inference. To achieve the latter, a longitudinal study is necessary. Finally, the inclusion of other dynamic sensorimotor tasks, such as gait, could further clarify the different aspects of sensorimotor control.

\section{CONCLUSIONS}

In the present study, patients with NSLBP showed alterations of cortical thickness in brain regions that play an important role in the cognitive regulation of pain, as well as an impaired STSTS performance compared with healthy controls. Cortical thickening was associated with increased pain intensity in the individuals with NSLBP. In addition, decreased cortical thickness of the rostral anterior cingulate cortex was correlated with lower STSTS performance on an unstable support surface. These findings suggest that in addition to measures of volume, cortical thickness may provide a more complete understanding of the central basis of sensorimotor tasks, more specifically in the NSLBP population.

\section{ACKNOWLEDGMENTS}

The authors are grateful to the study volunteers for their participation.

Disclosures: Madelon Pijnenburg—RELATED: Grant: Agency for Innovation by Science and Technology-Flanders, Comments: PhD fellowship: Lotte JanssensRELATED: Grant: Research Foundation Flanders, Comments: postdoctoral fellowship with grant number 12M9815N. Nina Goossens-RELATED: Grant: Agency for Innovation by Science and Technology-Flanders, Comments: PhD fellowship.

\section{REFERENCES}

1. Waddell G. 1987 Volvo award in clinical sciences: a new clinical model for the treatment of low-back pain. Spine (Phila $\mathrm{Pa} 1976$ ) 1987;12:632-44 CrossRef Medline

2. Airaksinen O, Brox JI, Cedraschi C, et al. Chapter 4: European guidelines for the management of chronic nonspecific low back pain. Eur Spine J 2006;15(suppl 2):S192-300 CrossRef Medline

3. Hancock MJ, Maher CG, Laslett M, et al. Discussion paper: what happened to the 'bio' in the bio-psycho-social model of low back pain? Eur Spine J 2011;20:2105-10 CrossRef Medline

4. Balagué F, Mannion AF, Pellisé F, et al. Non-specific low back pain. Lancet 2012;379:482-91 CrossRef Medline

5. Apkarian AV, Sosa Y, Sonty S, et al. Chronic back pain is associated with decreased prefrontal and thalamic gray matter density. $J \mathrm{Neu}$ rosci 2004;24:10410-15 CrossRef Medline

6. Schmidt-Wilcke T, Leinisch E, Gänssbauer S, et al. Affective components and intensity of pain correlate with structural differences in gray matter in chronic back pain patients. Pain 2006;125:89-97 CrossRef Medline

7. Ivo R, Nicklas A, Dargel J, et al. Brain structural and psychometric alterations in chronic low back pain. Eur Spine J 2013;22:1958-64 CrossRef Medline

8. Baliki MN, Schnitzer TJ, Bauer WR, et al. Brain morphological signatures for chronic pain. PLoS One 2011;6:e26010 CrossRef Medline

9. Mao C, Wei L, Zhang Q, et al. Differences in brain structure in patients with distinct sites of chronic pain: a voxel-based morphometric analysis. Neural Regen Res 2013;8:2981-90 CrossRef Medline

10. Brumagne S, Janssens L, Knapen S, et al. Persons with recurrent low back pain exhibit a rigid postural control strategy. Eur Spine J 2008; 17:1177-84 CrossRef Medline

11. Laird RA, Gilbert J, Kent P, et al. Comparing lumbo-pelvic kinematics in people with and without back pain: a systematic review and meta-analysis. BMC Musculoskelet Disord 2014;15:229 CrossRef Medline

12. Ruhe A, Fejer R, Walker B. Center of pressure excursion as a measure of balance performance in patients with non-specific low back pain compared to healthy controls: a systematic review of the literature. Eur Spine J 2011;20:358-68 CrossRef Medline

13. Claeys K, Dankaerts W, Janssens L, et al. Altered preparatory pelvic control during the sit-to-stance-to-sit movement in people with non-specific low back pain. J Electromyogr Kinesiol 2012;22:821-28 CrossRef Medline

14. Hall LM, Brauer S, Horak F, et al. Adaptive changes in anticipatory postural adjustments with novel and familiar postural supports. J Neurophysiol 2010;103:968-76 CrossRef Medline

15. Lyoo CH, Ryu YH, Lee MS. Cerebral cortical areas in which thickness correlates with severity of motor deficits of Parkinson's disease. J Neurol 2011;258:1871-76 CrossRef Medline

16. Panizzon MS, Fennema-Notestine C, Eyler LT, et al. Distinct genetic influences on cortical surface area and cortical thickness. Cereb Cortex 2009;19:2728-35 CrossRef Medline 
17. Winkler AM, Kochunov P, Blangero J, et al. Cortical thickness or grey matter volume? The importance of selecting the phenotype for imaging genetics studies. Neuroimage 2010;53:1135-46 CrossRef Medline

18. Rakic P. Specification of cerebral cortical areas. Science 1988;241: 170-76 CrossRef Medline

19. Wierenga LM, Langen M, Oranje B, et al. Unique developmental trajectories of cortical thickness and surface area. Neuroimage 2014; 87:120-26 CrossRef Medline

20. Rakic P. Defects of neuronal migration and the pathogenesis of cortical malformations. Prog Brain Res 1988;73:15-37 CrossRef Medline

21. Rakic P. Evolution of the neocortex: a perspective from developmental biology. Nat Rev Neurosci 2009;10:724-35 CrossRef Medline

22. Fischl B. FreeSurfer. Neuroimage 2012;62:774-81 CrossRef Medline

23. Kong J, Spaeth RB, Wey HY, et al. S1 is associated with chronic low back pain: a functional and structural MRI study. Mol Pain 2013; 9:43 CrossRef Medline

24. Dolman AJ, Loggia ML, Edwards RR, et al. Phenotype matters: the absence of a positive association between cortical thinning and chronic low back pain when controlling for salient clinical variables. Clin J Pain 2014;30:839-45 CrossRef Medline

25. Pijnenburg M, Caeyenberghs K, Janssens L, et al. Microstructural integrity of the superior cerebellar peduncle is associated with an impaired proprioceptive weighting capacity in individuals with non-specific low back pain. PLoS One 2014;9:e100666 CrossRef Medline

26. Pijnenburg M, Brumagne S, Caeyenberghs $K$, et al. Resting-state functional connectivity of the sensorimotor network in individuals with non-specific low back pain and the association with the sit-tostand-to-sit task. Brain Connect 2015;5:303-11 CrossRef Medline

27. Fairbank JC, Pynsent PB. The Oswestry Disability Index. Spine (Phila Pa 1976) 2000;25:2940-52; discussion 2952 CrossRef Medline

28. Vernon H, Mior S. The Neck Disability Index: a study of reliability and validity. J Manipulative Physiol Ther 1991;14:409-15 Medline

29. Jensen MP, Karoly P, Braver S. The measurement of clinical pain intensity: a comparison of six methods. Pain 1986;27:117-26 CrossRef Medline

30. Smeets RJ, Hijdra HJ, Kester AD, et al. The usability of six physical performance tasks in a rehabilitation population with chronic low back pain. Clin Rehabil 2006;20:989-97 CrossRef Medline

31. Simmonds MJ, Olson SL, Jones S, et al. Psychometric characteristics and clinical usefulness of physical performance tests in patients with low back pain. Spine (Phila Pa 1976) 1998;23:2412-21 CrossRef Medline

32. Lovibond PF, Lovibond SH. The structure of negative emotional states: comparison of the Depression Anxiety Stress Scales (DASS) with the Beck Depression and Anxiety Inventories. Behav Res Ther 1995;33:335-43 CrossRef Medline

33. Dale AM, Fischl B, Sereno MI. Cortical surface-based analysis, I: segmentation and surface reconstruction. Neuroimage 1999;9: 179-94 CrossRef Medline

34. Fischl B, Dale AM. Measuring the thickness of the human cerebral cortex from magnetic resonance images. Proc Natl Acad Sci U S A USA 2000;97:11050-55 CrossRef Medline

35. Fischl B, Liu A, Dale AM. Automated manifold surgery: constructing geometrically accurate and topologically correct models of the human cerebral cortex. IEEE Trans Med Imaging 2001;20:70-80 CrossRef Medline

36. Fischl B, van der Kouwe A, Destrieux C, et al. Automatically parcellating the human cerebral cortex. Cereb Cortex 2004;14:11-22 CrossRef Medline

37. Fischl B, Sereno MI, Dale AM. Cortical surface-based analysis, II: inflation, flattening, and a surface-based coordinate system. Neuroimage 1999;9:195-207 CrossRef Medline

38. Fischl B, Sereno MI, Tootell RB, et al. High-resolution intersubject averaging and a coordinate system for the cortical surface. Hum Brain Mapp 1999;8:272-84 Medline

39. Fischl B, Salat DH, van der Kouwe AJ, et al. Sequence-independent segmentation of magnetic resonance images. Neuroimage 2004; 23(suppl 1):S69-84 CrossRef Medline

40. Han X, Jovicich J, Salat D, et al. Reliability of MRI-derived measurements of human cerebral cortical thickness: the effects of field strength, scanner upgrade and manufacturer. Neuroimage 2006;32: 180-94 CrossRef Medline

41. Jovicich J, Czanner S, Greve D, et al. Reliability in multi-site structural MRI studies: effects of gradient non-linearity correction on phantom and human data. Neuroimage 2006;30:436-43 CrossRef Medline

42. Ségonne F, Dale AM, Busa E, et al. A hybrid approach to the skull stripping problem in MRI. Neuroimage 2004;22:1060-75 CrossRef Medline

43. Fischl B, Salat DH, Busa E, et al. Whole brain segmentation: automated labeling of neuroanatomical structures in the human brain. Neuron 2002;33:341-55 CrossRef Medline

44. Desikan RS, Ségonne F, Fischl B, et al. An automated labeling system for subdividing the human cerebral cortex on MRI scans into gyral based regions of interest. Neuroimage 2006;31:968-80 CrossRef Medline

45. Hoaglin DC, Inglewicz B. Fine tuning some resistant rules for outlier labeling. J Am Statist Ass 1987;82:1147-49 CrossRef

46. Pan PL, Zhong JG, Shang HF, et al. Quantitative meta-analysis of grey matter anomalies in neuropathic pain. Eur J Pain 2015;19: 1224-31 CrossRef Medline

47. Smallwood RD, Laird AR, Ramage AE, et al. Structural brain anomalies and chronic pain: a quantitative meta-analysis of gray matter volume. J Pain 2013;14:663-75 CrossRef Medline

48. Lerch JP, Evans AC. Cortical thickness analysis examined through power analysis and a population simulation. Neuroimage 2005;24: 163-73 CrossRef Medline

49. Lee MC, Tracey I. Imaging pain: a potent means for investigating pain mechanisms in patients. Br J Anaesth 2013;111:64-72 CrossRef Medline

50. Wager TD, Davidson ML, Hughes BL, et al. Prefrontal-subcortical pathways mediating successful emotion regulation. Neuron 2008; 59:1037-50 CrossRef Medline

51. Rocca MA, Ceccarelli A, Falini A, et al. Brain gray matter changes in migraine patients with T2-visible lesions: a 3-T MRI study. Stroke 2006;371765-70 CrossRef Medline

52. Valfrè W, Rainero I, Bergui M, et al. Voxel-based morphometry reveals gray matter abnormalities in migraine. Headache 2008;48: 109-17 Medline

53. Wiech K, Kalisch R, Weiskopf N, et al. Anterolateral prefrontal cortex mediates the analgesic effect of expected and perceived control over pain. J Neurosc 2006;26:11501-09 CrossRef Medline

54. Brody AL, Barsom MW, Bota RG, et al. Prefrontal-subcortical and limbic circuit mediation of major depressive disorder. Semin Clin Neuropsychiatry 2001;6:102-12 CrossRef Medline

55. Shiba Y, Santangelo AM, Roberts AC. Beyond the medial regions of prefrontal cortex in the regulation of fear and anxiety. Front Syst Neurosci 2016;10:12 CrossRef Medline

56. O'Sullivan K, Dankaerts W, O'Sullivan L, et al. Cognitive functional therapy for disabling nonspecific chronic low back pain: multiple case-cohort study. Phys Ther 2015;95:1478-88 CrossRef Medline

57. Jensen KB, Kosek E, Wicksell R, et al. Cognitive behavioral therapy increases pain-evoked activation of the prefrontal cortex in patients with fibromyalgia. Pain 2012;153:1495-503 CrossRef Medline

58. Seminowicz DA, Shpaner M, Keaser ML, et al. Cognitive-behavioral therapy increases prefrontal cortex gray matter in patients with chronic pain. J Pain 2013;14:1573-84 CrossRef Medline

59. Kiers $H$, Brumagne $S$, van Dieën J, et al. Ankle proprioception is not targeted by exercises on an unstable surface. Eur J Appl Physiol 2012; 112:1577-85 CrossRef Medline

60. Stedman TL. The American Heritage Stedman's Medical Dictionary. Boston: Houghton Mifflin Co; 2005 
61. Isableu B, Vuillerme N. Differential integration of kinaesthetic signals to postural control. Exp Brain Res 2006;174:763-68 CrossRef Medline

62. Claeys K, Brumagne S, Dankaerts W, et al. Decreased variability in postural control strategies in young people with non-specific low back pain is associated with altered proprioceptive reweighting. Eur J Appl Physiol 2011;111:115-23 CrossRef Medline

63. Lord SR. Aging and falls: causes and prevention. J Musculoskelet Neuronal Interact 2007;7:347 Medline
64. Peyron R, Laurent B, García-Larrea L. Functional imaging of brain responses to pain: a review and meta-analysis (2000). Neurophysiol Clin 2000;30:263-88 CrossRef Medline

65. May A. Chronic pain may change the structure of the brain. Pain 2008;137:7-15 CrossRef Medline

66. Schmidt-Wilcke T. Neuroimaging of chronic pain. Best Pract Res Clin Rheumatol 2015;29:29-41 CrossRef Medline

67. Rakic P. Radial unit hypothesis of neocortical expansion. Novartis Found Symp 2000;228:30-42; discussion 42-52 Medline 